\title{
ESTUDO DE CASO SOBRE O USO DA MODELAGEM MULTINÍVEL PARA A HARMONIZAÇÃO DE TERMINOLOGIAS DE ENFERMAGEM
}

A case study on the use of multilevel modeling for the harmonization of nursing terminologies.

\author{
Joyce Rocha de Matos Nogueira'; Timothy Wayne Cook ${ }^{2}$; Luciana Tricai Cavalini ${ }^{3}$
}

Resumo Objetivos: Este trabalho apresenta um caso de harmonização de terminologias de enfermagem seguida pela representação de conceitos clínicos de acordo com os princípios de modelagem multinível. Materiais e métodos: $O$ modelo Fehring de diagnóstico da diminuição do débito cardíaco baseado na terminologia da North American Nursing Diagnosis Association (NANDA) foi mapeado a termos da Classificação Internacional para a Prática de Enfermagem (CIPE). Os conceitos mapeados foram modelados como arquétipos e Concept Constraint Definitions (CCDs), de acordo, respectivamente, com as especificações openEHR e MLHIM. Resultados: Apesar de o mapeamento entre as terminologias resultar incompleto, todos os conceitos do modelo Fehring foram modelados em arquétipos e CCDs. Conclusão: Este trabalho demonstrou a capacidade da modelagem multinível de harmonizar terminologias de enfermagem que não são totalmente compatíveis.

Palavras-chave: Terminologia como Assunto, Registros Eletrônicos de Saúde, Informática em Enfermagem

Abstract Aims: This paper presents a case for harmonization of nursing terminologies followed by the representation of clinical concepts in accordance with the principles of multilevel modeling. Materials and Methods: The Fehring model for the diagnosis of decreased cardiac output based on the terminology of the North American Nursing Diagnosis Association (NANDA) was mapped to the terms of the International Classification for Nursing Practice (ICNP). The concepts were mapped and modeled as archetypesandConcept Constraint Definitions (CCDs), in accordance, respectively, to the MLHIM and openEHR specifications. Results: Despite the incompleteness of the resulting terminology mapping, all the Fehring model concepts were modeled in archetypes and CCDs. Conclusion: This study demonstrated the ability of multilevel modeling to harmonize nursing terminologies that are not fully compatible.

Keywords: Terminology as Topic, Electronic Health Records, Nursing Informatics

\footnotetext{
1. Bolsista de Nível Superior do Programa de Apoio Técnico às Atividades de Ensino, Pesquisa e Extensão (PROATEC) da Universidade do Estado do Rio de Janeiro - Mestrado em Saúde Coletiva Pela Universidade Federal Fluminense (2013). 2. Consultor - Mestrado em Health Informatics pela University of Central Lancashire, Inglaterra(2006) 3. Professor Adjunto da Universidade do Estado do Rio de Janeiro, Brasil - Pós-doutorado em Medicina Social pelo Karolinska Institutet, Suécia (2008).
} 


\section{Introdução}

A informática em enfermagem é definida como a área do conhecimento que estuda a aplicação de recursos tecnológicos no ensino, prática, gestão e cuidado de enfermagem'. Sistemas de informação em enfermagem devem considerar as necessidades específicas da área, tais como a padronização de conceitos referentes ao processo de trabalho da enfermagem. Em geral, os esforços nesta direção se concentram no desenvolvimento de uma terminologia de referência, edificada sobre a interface entre terminologias e tabelas já existentes, sejam elas específicas da área de enfermagem ou gerais da área da saúde².

Este esforço de padronização com base no desenvolvimento de vocabulários controlados teve como consequência a criação de uma multiplicidade de terminologias, desenvolvidas de forma independente por diversas instituições, sem que houvesse, necessariamente, o estabelecimento de acordos entre eles ${ }^{3}$. Assim, a harmonização de terminologias, entendida como um processo de consenso terminológico, é um requisito importante para garantir que os conceitos de enfermagem sejam representados de uma maneira que facilite sua utilização em todos os níveis de atenção e gestão. Entretanto, há poucas iniciativas nesse sentido e, dado o crescimento da área de informática em enfermagem, esta poderia seguir o mesmo caminho da ausência de interoperabilidade que ocorre com sistemas médicos, o que não é desejado. As ideias mais prevalentes para a solução desta questão sugerem a criação de um sistema de informação de saúde com um modelo de dados compartilhado, ou seja, a definição de um consenso sobre a forma de modelar os bancos de dados ou pelo menos os extratos de informação a serem compartilhadas entre os sistemas".

No entanto, a complexa dinâmica do sistema de saúde não permite a construção de sistemas monolíticos ou mesmo terminologias totalmente abrangentes, como pode ser observado sobre os esforços de desenvolvimen- to da Standardized Nomenclature of Medicine-Clinical Terms (SNOMED-CT) e as iniciativas para a sua harmonização com outras terminologias, incluindo a Classificação Internacional da Prática de Enfermagem (CIPE)5. Portanto, há a necessidade de um novo olhar sobre as questões de implementação prática de sistemas de informação em enfermagem. Neste sentido, o objetivo deste trabalho é apresentar um estudo de caso de harmonização de terminologias de enfermagem seguido pela representação de conceitos clínicos de acordo com os princípios de modelagem multinível, entendido como um método de desenvolvimento de sistemas de informação em saúde semanticamente interoperáveis, em função da definição de um modelo de informação independente da camada de modelagem do conhecimento ${ }^{6}$.

\section{Métodos}

Para este estudo de caso, selecionaram-se as variáveis (ou seja, as características dos pacientes) que definem o diagnóstico de diminuição do débito cardíaco, validadas de acordo com a metodologia definida por Fehring para o diagnóstico de enfermagem (1987) ${ }^{7}$. Esta iniciativa de validação foi implementada pela North American Nursing Diagnosis Association (NANDA), que desenvolve uma terminologia de enfermagem amplamente utilizada em termos globais (NANDA-I). Os conceitos clínicos de diminuição do débito cardíaco foram validados de acordo com o modelo Fehring por Martins et al. ${ }^{8}$, sendo um dos poucos artigos na literatura que descrevem de forma detalhada o processo de validação utilizado na definição de uma diagnóstico de enfermagem. No presente estudo, os mesmos foram utilizados para a busca de termos correspondentes na CIPE versão 2, através do ambiente web de busca ICNP, o C-Space. Para cada conceito do modelo Fehring, foram elaborados modelos de representação do conhecimento correspondente. 
A representação do conhecimento baseou-se nos princípios de modelagem multinível de sistemas de informação de saúde. Nesta abordagem, há uma separação entre a camada de desenvolvimento de software, representada pelo Modelo de Referência, e o Modelo de Dados, representado pelo Modelo de Domínio9. A ideia subjacente à modelagem multinível é de que mudanças na estrutura da informação se refletem apenas no Modelo de Domínio, o que reduz as demandas por mudanças nos mecanismos de persistência de sistemas de informação. Além disso, o conhecimento é modelado diretamente pelos próprios especialistas do domínio, o que evita a necessidade de interpretação dos conceitos do domínio através de uma interação ad hoc entre o especialista da área de saúde e os desenvolvedores de aplicativos ${ }^{10}$.

A primeira etapa da modelagem do conhecimento consistiu na busca de arquétipos openEHR9 no repositório web de arquétipos Clinical Knowledge Manager(CKM). Posteriormente, os mesmos conceitos clínicos foram modelados como Concept Constraint Definitions(CCDs), de acordo com as especificações Multilevel Healthcare Information Modeling(MLHIM) versão 2.3.08, através do mapeamento gráfico dos conceitos clínicos pelo Constraint Definition Designer(CDD), uma ferramenta de representação gráfica do Modelo de Referência MLHIM no formato de mapas mentais(código aberto disponível em https://launchpad.net/mlhim).

\section{Resultados}

A análise do modelo Fehring de validação clínica do diagnóstico de diminuição do débito cardíaco resultou na identificação de 15 conceitos: Dispneia, Fadiga, Edema, Ortopneia, Dispneia paroxística noturna, Pressão venosa central elevada, Ganho de peso, Hepatomegalia, Distensão da veia jugular, Palpitações, Crepitações, Oligúria, Tosse, Pele úmida e Alterações da cor da pele.

Dos conceitos incluídos, oito deles apresentaram termos correspondentes na CIPE: Dispneia, Fadiga, Edema, Ortopneia, Dispneia paroxística noturna, Ganho de peso, Tosse e Pele úmida. Para os sete conceitos restantes (Pressão venosa central elevada, Hepatomegalia, Distensão da veia jugular, Palpitações, Crepitações, Oligúria, e Alterações da cor da pele), não foram encontrados termos equivalentes na CIPE, resultando em uma cobertura de termos pela CIPE de 53,3\%. Por outro lado, a busca no CKM resultou na obtenção de nove arquétipos openEHR para todas as características do diagnóstico de diminuição do débito cardíaco de acordo com a validação do modelo de Fehring, sendo que dois deles encontravam-se validados e sete em diferentes momentos do processo de validação (Tabela 1).

Tabela 1:

A linha de Mapeamento entre conceitos clínicos da diminuição do débito cardíaco segundo o modelo Fehring, os termos CIPE e os arquétipos openEHR.

\begin{tabular}{|c|c|c|c|c|c|}
\hline \multirow[t]{2}{*}{ Conceito } & \multicolumn{3}{|c|}{ Mapeamento para a CIPE } & \multicolumn{2}{|c|}{ Arquétipo openEHR } \\
\hline & Termo & Eixo & Código & Nome & Classe \\
\hline Dispneia & Dispneia & Foco & 10006461 & Symptom** & CLUSTER \\
\hline Fadiga & Fadiga & Foco & 10007717 & Symptom** & CLUSTER \\
\hline Edema & Oedema & Foco & 10013627 & Oedema** & CLUSTER \\
\hline Ortopneia & Ortopneia & Foco & 10013823 & Respirations* & OBSERVATION \\
\hline Dispneia paroxística noturna & Não há & - & - & Respirations* & OBSERVATION \\
\hline Pressão venosa central elevada & Não há & - & - & Central venous pressure ${ }^{* *}$ & OBSERVATION \\
\hline Ganho de peso & Não há & - & - & Body weight* & OBSERVATION \\
\hline Hepatomegalia & Não há & - & - & Examination of the abdomen** & CLUSTER \\
\hline Distensão da veia jugular & Não há & - & - & Physical examination findings** & OBSERVATION \\
\hline Palpitações & Não há & - & - & Pulse $e^{* * *}$ & OBSERVATION \\
\hline Crepitações & Não há & - & - & Respirations* & OBSERVATION \\
\hline Oligúria & Não há & - & - & Symptom** & CLUSTER \\
\hline Tosse & Coughing & Foco & 10005249 & Symptom** & CLUSTER \\
\hline Pele úmida & Clammy skin & Foco & 10012149 & Inspection of skin** & CLUSTER \\
\hline Alterações da cor da pele & Não há & - & - & Inspection of skin** & CLUSTER \\
\hline
\end{tabular}

*Arquétipo em estágio“Published” (validado). ** Arquétipo em estágio“Draft” (não validado). *** Arquétipo em estágio“Team review” (não validado). 
Observou-se, adicionalmente, que a correspondência exata entre os conceitos do modelo Fehring e os arquétipos openEHR somente seria possível se os arquétipos openEHR correspondentes fossem especializados ou restritos em nível de template, o que adicionou uma complexidade adicional ao processo de representação do conhecimento referente aos conceitos modelados no presente estudo.

Por exemplo, para a representação do conceito de Dispneia, em nível de template openEHR ${ }^{12}$, para o arquétipo openEHR "Symptom", foram expressos os seguintes componentes: o ELEMENT "Symptom" [at0001], com a inclusão de uma terminologia interna para tornar este ELEMENT invariante no valor “Dispneia”, e o ELEMENT "Nil significant" [at0002], cujo valor TRUE corresponde à ausência de dispneia. Essa representação apresenta um nível importante de complexidade em termos de implementação prática em aplicativos. Além disso, a necessidade de se utilizar um ELEMENT Booleano cujo valor TRUE corresponde a uma negativa pode trazer dificuldades de interpretação do conceito para o usuário de um sistema baseado em tal restrição ao arquétipo openEHR para o conceito "Symptom".

Entretanto, quando os mesmos conceitos do modelo Fehring foram modelados como CCDs MLHIM, todos pu- deram ser modelados diretamente, visto que, em MLHIM, não há correspondência biunívoca entre um conceito clínico e sua representação como $C C D^{11}$. Assim, todos os conceitos as características do diagnóstico de diminuição do débito cardíaco, de acordo com a validação do modelo de Fehring, foram modelados como Pluggable Complex Types (PCTs) (Cavalini e Cook, 2012) que podem ser recombinados em quaisquer tipos de CCDs. A representação gráfica do modelo abstrato de um PCT modelado para dispneia é mostrado na Figura 1.

\section{Discussão}

Este artigo apresentou um processo de harmonização de terminologias de enfermagem e representação de seus conceitos para duas especificações de modelagem multinível (openEHR e MLHIM). Nossos resultados demonstraram a viabilidade da representação do conhecimento padronizado de conceitos de enfermagem através da adoção da metodologia de modelagem multinível de sistemas de informação de saúde, como uma estratégia eficiente de harmonização de terminologias ${ }^{13}$.

Assim, a adoção dos modelos de representação do conhecimento de acordo com os princípios de

\section{Figura 1:}

Representação Gráfica do modelo abstrado do PCT MLHIM para o conceito clínico de dispneia, de acordo com o modelo Fehring. maxorcurs 9 minOccurs *
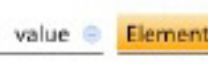

maxoccurs $=1$

feeder_audit $\$$ 
modelagem multinível pode atuar como um processo mediador de harmonização de terminologias de enfermagem. Ou seja, os modelos multinível de representação de conhecimento podem ser utilizados para modelar termos de quaisquer vocabulários controlados, sendo que extratos de informação gerados a partir destes modelos são semanticamente válidos em outros sistemas de informação, mesmo que eles adotem outras terminologias ${ }^{13}$.

Como observado no presente trabalho, não houve correspondência dos termos das terminologias NANDA e CIPE mesmo para um pequeno conjunto de conceitos, e os processos de negociação de harmonização de terminologias são, por definição, longos e dispendiosos, visto serem baseados em estratégias de consenso ${ }^{14}$. Por sua vez, ambas as especificações de modelagem multinível utilizadas neste estudo (openEHR e MLHIM) foram capazes de representar todos os conceitos as características do diagnóstico de diminuição do débito cardíaco de acordo com a validação do modelo de Fehring, embora as características técnicas do Modelo de Arquétipos das especificações openEHR tornem seu processo mais complexo, tanto em termos de modelagem, como em termos de implementação de aplicativos, em comparação com a modelagem de CCDs MLHIM. Esta limitação para a implementação prática de sistemas de informação em saúde baseados no modelo openEHR já foi descrita anteriormente por outros autores ${ }^{15}$.

Independentemente da especificação a ser adotada, este estudo sugere que a modelagem multinível é uma alternativa viável para a representação do conhecimento em enfermagem, de forma colaborativa com o desenvolvimento de terminologias de referência e vocabulários controlados. É importante notar, contudo, que a análise aqui apresentada, para um conceito específico da área de enfermagem, pode ser estendida para todas as outras áreas e profissões da saúde, dado que a modelagem multinível foi concebida para a modelagem de ecossistemas de informação em saúde semanticamente interoperáveis, o que é um requisito do setor ${ }^{16}$.

\section{Conclusão}

O presente estudo demonstrou a viabilidade de utilização da modelagem multinível, em ambas as especificações existentes (openEHR e MLHIM), para a mediação entre diferentes terminologias de enfermagem. O processo de harmonização de terminologias, entretanto, é tarefa complexa, visto que o domínio da saúde é consideravelmente vasto em termos de número de conceitos e sua dinâmica espaço-temporal.

O tema da representação do conhecimento em enfermagem, para a obtenção de interoperabilidade dos registros eletrônicos de saúde, apresenta uma complexidade significativa. Em função disto, o campo da informática de enfermagem provavelmente irá continuar a expandir a inclusão de seus especialistas, de forma a permitir o diálogo com pesquisadores interdisciplinares. Assim, será possível alavancar o conhecimento adquirido a partir dessas trocas de conhecimento sem, contudo, deixar de representar o conceito específico de enfermagem nos sistemas de informação em saúde.

Como visto no presente estudo, há ainda muitos desafios que precisam ser enfrentados no campo da informática em enfermagem. Destaca-se, de forma especial, a necessidade de integrar a representação do conhecimento em enfermagem às terminologias padronizadas da área. Dessa forma, a tecnologia da informação pode ser um poderoso instrumento de apoio à prática da profissão de enfermagem. 


\section{Agradecimentos}

Este trabalho é um produto do Laboratório"Multilevel Healthcare Information Modeling"(LA-MLHIM), associado ao Instituto Nacional de Ciência e Tecnologia - Medicina Assistida por Computação Científica (INCT-MACC) financiamento CNPq e Faperj.

\section{Referências}

1. Staggers $N$, Thompson CB. The evolution of definitions for nursing informatics: a critical analysis and revised definition. J. Am. Med. Inform. Assoc. 2002; 9(3):255-61.

2. Coenen A, Marin HF, Park H,Bakken S. Collaborative efforts for representing nursing concepts in computer-based systems: international perspectives. J. Am. Med. Inform. Assoc. 2001;8(3):202-11.

3. Smith B, Brochhausen M. Putting biomedical ontologies to work. Methods Inf Med. 2010; 49(2): 135-40

4. Hayrinen $\mathrm{K}$, Saranto $\mathrm{K}$. The use of nursing terminology in electronic documentation. Stud. Health Technol. Inform. 2009; 146:342-6.

5. Park HA, Lundberg C, Coenen A,Konicek D. Evaluation of the content coverage of SNOMED CT representing ICNP seven-axis version 1 concepts. Methods Inf. Med. 2011;50(5):472-8.
6. Cavalini LT, Cook TW. Engenharia do conhecimento de sistemas de informação em saúde com base em modelos multinível minimalistas. J Bras Tele. 2012; 1(2):43-50.

7. Fehring R. Methods to validate nursing diagnoses. Heart \& Lung. 1987; 16(6), 625-9. 8. Martins QC, Aliti G, Rabelo ER. Decreased cardiac output: clinical validation in patients with decompensated heart failure. Int. J. Nurs. Terminol. Classif. 2010; 21(4):156-65.

9. Beale T. Archetypes and the EHR. Stud. Health Technol. Inform. 2003; 96:238-44.

10. Garde S, Hovenga E, Buck J, Knaup P. Expressing clinical data sets with openEHR archetypes: a solid basis for ubiquitous computing. Int. J. Med. Inform. 2007;76(Suppl 3):S334-41.

11. Cavalini LT, Cook TW. Health informatics: the relevance of open source and multilevel modeling. IFIP Adv. Inform. Commun. Technol. 2011; 365:338-47.

12. Beale T, Heard S. The Template Object Model (TOM). London: openEHR Foundation; 2007.

13. Sundvall E, Qamar R, Nyström M, Forss M, Petersson H, Karlsson D, Ahlfeldt H, Rector A. Integration of tools for binding archetypes to SNOMED CT. BMC Med. Inform. Decis. Mak. 2008; 8(Suppl 1):S7.

14. Martinez MN, Lindquist $D$, Modric S. Terminology challenges: defining modified release dosage forms in veterinary medicine. J. Pharm. Sci. 2010; 99(8):3281-90.

15. Kashfi H, Torgersson O. A migration to an openEHR-based clinical application. Stud. Health Technol. Inform. 2009; 150:152-6.

16. Kalra D, Tapuria A, Austin T, De Moor G. Quality requirements for EHR archetypes. Stud Health Technol Inform. 2012; 180: 48-52. 